\title{
Large values of Dirichlet polynomials, III
}

by

\author{
M. N. FUxrax (Cardiff)
}

1. Introduction. This paper continues [3] and. [4]. Our first object is to estimate the number of times that a Dirichlet polynomial is large. In [3] we showed that the fate of any Dirichlet polynomial of length. $N$ was entwined, with that of a standard Dirichlet polynomial of the conjugate length $D / N$. The question of how often two Dirichlet polynomials can be large simultaneously is important in Halász's method. In this case the two Dirichlet polynomials are of different lengths and we multiply them together. Multiplying together two Dirichlet polynomials of the same length merely estimates the number of times the square of either could be large, and so gives trivial bounds.

Our Theorem 1 generalizes the key estimate of [2] to Dirichlet polynomials containing a variable Dirichlet character. Theorem 2 contains further results and Theorem 3 is the appropriate application of Theorems 1. and 2 to our second object, which is to estimate the number of zeros of Dirichlet $L$-functions. In the usual notation. (see [6]) Theorem 3 implies

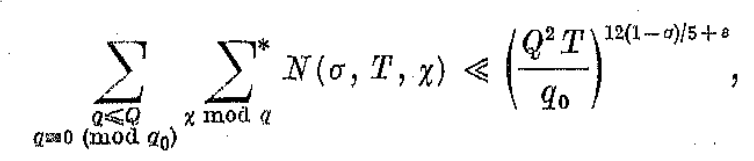

and for $\sigma>4 / 5$ only

$$
N(\dot{\sigma}, T) \ll T^{2(1-\sigma)+3} .
$$

Theorem 12.1 of $[6]$ contains (1.1) with $5 / 2$ in place of $12 / 5$ in the exponent. Forti and Viola [1.] improved. $5 / 2$ to $2.463 \ldots$, Jutila [5] to $2.460 \ldots$ and the author [4] to $2.4: 32 \ldots$ (Jutila anctually treats all characters to a fixed modulus, which is essentially the special case $Q=q_{0}$, where $q_{0}$ is a divisor of the given modulus.) The estimate (1.2) is the 'density hypothesis' and has a longer history. It is obtained in [1] for $\sigma>0.8059 \ldots$, and in [4] for $\sigma>0.801 .$.

This paper is based on [3], but supersedes neither [3] nor [4], as it provides an alternative way of using the reflection argument of [3]. 
When $\log N / \log D$ is close to a rational number with small denominatior it may be better to apply Lemma 2 several times as in [3] and then to form a product polynomial. The results of [3], [4] and the present pajer cannot be subsumed under one simple bound. For instance, an improve ment in the upper bound for $\zeta\left(\frac{1}{2}+i t\right)$ would improve the abscissan of validity of (1.2) found in [1] from 0.80 . to one smaller than $4 / 5$. The proof of (1.2) for $\sigma>4 / 5$ which is completed in the present paper wes only averages of $\zeta\left(\frac{1}{2}+i t\right)$.

2. Statement of the results. We consider Dirichlet polynomials of the form

$$
T(s, \chi)=\sum_{N+1}^{2 N} a(m) \chi(m) m^{-8}
$$

where $\chi$ is a Dirichlet character to some modulus $q, s=\sigma+i t$ is a complex. variable; a general Dirichlet polynomial can be partitioned intio sums of the form (2.1). Let $q_{0}$ be a positive integer, $Q \geqslant q_{0}$ and $T \geqslant 1$ be real numbers. Let

$$
\text { (2.2) } \quad D=Q^{2} T / q_{0}, \quad l=\log D
$$

We shall assume $D$ is large. Let $U$ be an aggregate of pairs $(s, \chi)$ satisfying

$$
\begin{gathered}
x \text { proper mod } q, \quad q \leqslant Q, \quad q=0\left(\bmod q_{0}\right), \\
0 \leqslant \sigma \leqslant l^{-1}, \\
\quad\left|t_{1}-t_{2}\right| \leqslant T, \\
\left|t_{1}-t_{2}\right| \geqslant 1 \text { when } \chi_{1}=\chi_{2} .
\end{gathered}
$$

Let $R$ be the cardinality of $U$. The first object of this paper is to obtain bounds for $R$ under the assumption

$$
|F(s, \chi)| \geqslant V
$$

for each pair $(s, \chi)$ of $U$. Let $B(M, U)$ be the least positive $B$ for which

$$
\sum_{(s, x) \in U}|F(s, \chi)| \leqslant\left\{\begin{array}{lll}
G^{u / 2} B & \text { if } & N \leqslant M \\
(N / M)^{9 / 2} G^{u / 2} B & \text { if } \quad M K \leqslant N D
\end{array}\right.
$$

where

$$
Q=\sum_{N+1}^{2 N}\left|a_{(}(m)\right|^{2}
$$

for every choice of coefficients $a(m)$, and let

$$
\text { (2.10) } \quad B(R, M, D)=\max B(M, U)
$$

taken over sets $U$ of cardinality $R$ with fixed $D$. We also define $B^{*}(M, \sigma)$

as the least positive $B$ for which

$$
\sum_{(s, \chi) \in U}|F(s, \chi)| \leqslant\left\{\begin{array}{lll}
M^{1 / 2} B & \text { if } & N \leqslant M, \\
M^{-1} N^{3 / 2} B & \text { if } & M \leqslant N \leqslant D
\end{array}\right.
$$

for every choice of coefficients $a(m)$ with $|a(m)| \leqslant 1$. Olearly

$$
B^{*}(M, U) \ll B(M, U)
$$

ret

$$
\Delta_{k:}=\max _{\alpha \leqslant D} d_{l}(q)
$$

be the maximum of the kth divisor function. We shall assume $D$ to be large and

$$
\Delta_{k} \ll D^{8}
$$

for any $\varepsilon>0$,

Since (2.7) implies that the left hand side of $(2.8)$ is at least $R V$, a bound for $B$ gives a bound for $R$ in terms of $V$.

The main result of [3] can be stated as follows

Limma 1. For $D>N$ we have

$$
B(R, N, D) \ll R^{1 / 2} N^{1 / 2} \log l+R D^{1 / 4} l
$$

and for any positive integer $k$

(2.16) $\quad B(R, N, D) \ll R^{1 / 2} N^{1 / 2} \log l+R N^{1 / 4} l^{2}+$

$$
+k^{k / 2} R^{1-1 / 2 \hbar k} N^{1 / 4} \Delta_{3}^{1 / 2}\left\{B\left(R, D^{k} / N^{k}, D\right)\right\}^{1 / 2 k t} l^{2+k / 4}
$$

In any case

$$
B(R, N, D) \ll R^{1 / 2}\left(D^{1 / 2}+-N^{1 / 2}\right) l .
$$

The implied oonstants are absolute.

An examination of the proof of lemma 1 leads us to the following restatement of it. Let $V(h, \omega)$ be any aggregate of ordered pairs $(s, \psi)$ with $\psi$ proper mod $f, f \leqslant Q / h, f=0\left(\bmod g_{0} /\left(h, q_{0}\right)\right)$ for which $\{(s, \omega \psi)\}$ is a subset of $U$.

In the notation of [3] our $q, x, f, \psi, h, \omega$ are $q_{1}, x_{1}, f_{1}, \psi_{1}, h_{1}$, and $\chi_{2} \bar{\psi}_{2}$. Wo oun sharpen (2.16) to

Limina 2. Let $\%$ be any positive integer and $D \geqslant N$. Then

(2.18) $\quad B(N, U) \ll R^{1 / 2} N^{1 / 2} \log l+R N^{1 / 4} l^{2}+$

$$
+k^{\gamma / 2} R^{1-1 / 2 / c} N^{1 / 4} \Delta_{3}^{1 / 2} 2^{2+1 k / 4} \max \left\{B\left(D^{k} / N^{k}, \sigma(h, \omega)\right)\right\}^{1 / 2 k}
$$


Moreover $B\left(D^{k} / N^{k}, U(h, \omega)\right)$ may be replaced by

$$
\Delta_{k} B^{*}\left(D^{k} / N^{k}, U(h, \omega)\right) \text {. }
$$

The simplest result we obtain from Lemma 2 is as follows.

Treorem 1 . In the notation above

$$
R \ll G V^{-2} N \log ^{2} l+G^{3} V^{-6} N D \Delta_{2}^{1 / 2} \Delta_{3} l^{11}
$$

provided that

$$
V>c_{1} G^{1 / 2} N^{1 / 4} l^{2}
$$

where $c_{1}$ is an absolute constant. Moreover for $N \leqslant D$

(2.22) $\quad B(N, U) \ll R^{1 / 2} N^{1 / 2} l \log l+R N^{1 / 4} l^{2}+R^{5 / 6} N^{1 / 6} A_{2}^{1 / 12} \Delta_{3}^{1 / 6} D^{1 / 6} l^{3}$.

Apart from divisor functions and logarithms, (2.20) contains (2.9) of [2] as a special case and enables a similar zero density theorem

$$
\sum_{q \leqslant Q} \sum_{x \bmod q}^{*} N(\sigma, T, \chi) \ll \max \left\{D^{3(1-q)(3 \sigma-1)+a}, D^{2(1-\sigma)+\varepsilon}\right\}
$$

to be deduced by the methods of [2]. This establishes (1.1) for $\sigma>3 / 4$, and the range $1 / 2 \leqslant \sigma \leqslant 3 / 4$ follows from Theorem 12.1 of [6].

To establish zero density theorems by the Flalasz-MontgomeryJutila method we need information about short sums $\left(N \leqslant D^{1 / r}\right)$. Applying Theorem 1 to $F^{r}(s, \chi)$ raises $N$ and $V$ to the $r$ th power and replaces $G$ by $\Delta_{r} G^{r}$ (see Lemma 3). If $V$ is somewhat larger than the bound (2.21) we can do better.

THEOREM 2. Let $p, q, r$ be positive integers such that $p \leqslant r-1$ and

$$
N^{r l-p} \geqslant D^{q-1}
$$

\section{Then}

$(2,25) \quad B(N, U) \ll R^{1-1 / 2 r} N^{1 / 2} \Delta_{2}+R \Delta_{2}\left(R^{-1} N^{p} D^{q}\right)^{1 /(4 q r+2 p)}+$

$$
+R N^{1 / 6} \Delta_{2}\left(R^{-1} D^{q+1}\right)^{1 /(22 q r+6 p)}+R A_{2}\left(N^{q r+p} D^{q}\right)^{1 /(8 q r+1-4 p)} \text {. }
$$

Moreover for

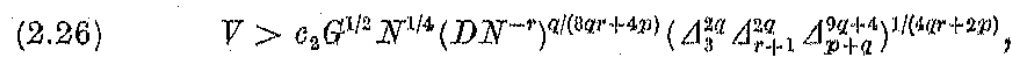

where $c_{2}$ depends only on $p, q$, and $r$, we have

(2.27) $R \ll\left(G N V^{-2}\right)^{r} \cdot D^{s}+\left(G V^{-2}\right)^{2 q r+p} N^{p} D^{2+8}+\left(G^{3} N V^{-6}\right)^{2 a r+p} D^{q+2+*}$.

The implied oonstants depend only on $p, q$, and in (2.27) also on $\varepsilon$.

We summarize the application of Theorems 1 and 2 to zero-density theorems as follows.
Trmomem 3. Suppose $|F(s, x)| \geqslant V$ at each pair $(s, \chi)$ of $U$ and for some integer $r \geqslant 2$

$$
(2.28) \quad N^{r} \leqslant D<N^{r+1} \text {. }
$$

Let

$$
V G^{-1 / 2}=N^{a-1 / 2}
$$

and $a>3 / 4$. Then for any $\varepsilon>0$ and $D$ sufficiently large

$$
\text { (2.30) } \quad R \ll D^{12(1-\alpha) / 5+8} \text {, }
$$

the constant depending on $r, a$ and $\varepsilon$. Moreover

$$
\text { (2.31) } \quad R \ll D^{2(1-a)+s}
$$

for

$$
\alpha>(3 r-1) /(4 r-2),
$$

the constant depending on $r, \alpha$ and $\varepsilon$. If the character $\chi$ is the same in all pairs $(s, \chi)$ and if for some integer $r \geqslant 2$

$$
N^{*} \leqslant T<N^{r+1}
$$

then

$$
R \ll T^{2(1-\alpha)+8}
$$

holds for

$$
\alpha> \begin{cases}4 / 5 & \text { if } r=2, \\ 7(3 r-1) /(28 r-12) & \text { if } \quad r \geqslant 3 .\end{cases}
$$

If in Sections 6 to 8 of [3] we replace the use of Theorem 1 by (2.31) and Theorem 2 by $(2.34)$ in the estimation of class $(i, n)$ zeros, we obtain (1.1.) for $\sigma>3 / 4$ and (1.2) for $\sigma>4 / 5$.

3. Proof of Theorem 1. We begin with a technical lemma. Lamma 3 . Fror $i=0,1, \ldots$, to let

$$
\mathscr{I}_{t}(s, \chi)=\sum_{1}^{M_{i}} a_{i}(m) \chi(m) m^{-s}
$$

with

$$
F_{0}(s, \chi)=\prod_{1}^{b} F_{i}(s, \chi)
$$

be Diriohlet polynomials. Tet

$$
Q_{i}=\sum_{i}^{M_{i}}\left|a_{i}(m)\right|^{2}
$$


Then if $M_{0} \leqslant D$ we have

$$
G_{0} \leqslant \Delta_{k} \prod_{1}^{k} G_{i}
$$

where $\Delta_{k}$ was defined by (2.13). If $M_{0} \leqslant D^{r}$, where $r>1$, then

$$
G_{0} \ll A_{k}^{r} \prod_{1}^{k} G_{i},
$$

where the constant depends on to and $r$.

Proof. We have

$$
\begin{aligned}
G_{0} & =\sum_{m}\left|\sum_{m_{1} \ldots m_{k}=m} a_{1}\left(m_{1}\right) \ldots a_{k_{k}}\left(m_{k_{k}}\right)\right|^{2} \\
& \leqslant \sum_{m} d_{k_{k}}(m) \sum_{m_{1} \ldots m_{k_{k}=m}=a_{1}}\left|a_{1}\left(m m_{1}\right)\right|^{2} \ldots\left|a_{k_{k}}\left(m_{r_{k}}\right)\right|^{2},
\end{aligned}
$$

which proves (3.4) at once, and (3.5) when we observe that

$$
d_{k}(m) \ll \Delta_{k}^{r}
$$

for $m \leqslant D^{\prime \prime}$

We now proceed to Theorem 1 . Take the $h$ and $w$ for which the maximum in (2.18) is attained, and replace $U$ by the corresponding subset $\{(s, \omega \psi)\}$. The bound $B^{*}(D / N, U(h, \omega))$ is attained by some Dirichlet polynomial $F_{1}(s, \psi)$ of length $M$ say. We consides the Dirichlet polynomial

$$
F_{0}(s, \psi)=F_{1}(s, \psi) F(s, \omega \psi) .
$$

In the notation of Lemma 3

$$
G_{0} \ll \Delta_{2} M G
$$

if $M \leqslant D / 4 N$ and

$$
G_{0} \ll \Delta_{2} M(M N / D)^{1 / 4} G
$$

if $M>D / 4 . N$, by (3.5) and the estimate (2.14). For simplicity we shall suppose $A \leqslant D / 4 . N$. Both cases give the same upper bound apart from the constant.

If $(2.7)$ holds at each $(s, x)$ we have by Lemirnal 2 ,

$$
\begin{aligned}
V \sum\left|H_{1}(s, \psi)\right| & \ll\left(\Delta_{2} M G\right)^{1 / 2} B(\Delta \cdot M N, U(h, \omega)) \\
& \ll G^{1 / 2} M^{1 / 2} \Delta_{2}^{1 / 2} R^{1 / 2} D^{1 / 2} l,
\end{aligned}
$$

where we have used (2.17). We deduce that

$$
B^{*}(D / N, U(h, \omega)) \ll G^{1 / 2} V^{-1} R^{1 / 2} D^{1 / 2} \Delta_{2}^{1 / 2} l^{1 / 2} .
$$

Substituting (3.12) into Lemma 2 we have

$$
\begin{aligned}
& \sum\left|F^{\prime}(s, x)\right| \\
& \ll G^{1 / 2}\left\{R^{1 / 2} N^{1 / 2} \log l+R N^{1 / 4} \eta^{2}+G^{1 / 4} R^{3 / 4} V^{-1 / 2} N^{1 / 4} D^{1 / 4} \Delta_{2}^{1 / 4} \Delta_{3}^{1 / 2} \eta^{11 / 4}\right\} .
\end{aligned}
$$

Fence if (2.21) holds with a sufficiently large $o_{1}$, the coefficient of $R$ on the left of (3.13) is at least twice that on the right, and we have

$$
R \ll G V^{-2} N \log ^{2} l+G^{3} V^{-6} N D \Delta_{2}^{\mathrm{I} / 2} \Delta_{3} l^{11},
$$

which proves (2.20). The assertion (2.22) follows by dividing the set $U$ up according to

(3.15) $\quad V \leqslant|F(s, x)|<2 \nabla$

and summing over a geometric progression of values of $V$.

4. Proof of Theorem 2. We work with powers of $F(s, \chi)$. Let $F_{1}(s, y)$ of length $M$ be extremal for $B^{*}\left(D / N^{r}, U(h, \omega)\right)$. We consider .

$$
F_{0}(s, \chi)=\mathbb{H}^{p}(s, \chi) \dot{F}_{1}^{\alpha}(s, \chi)
$$

where $p$ and $q$ are positive integers, $p \leqslant r-1$, with

$$
N^{p}\left(D / N^{r}\right)^{q} \leqslant D
$$

As in the proof of Theorem 1 there are two cases. We shall treat the ease

$$
2^{n+a} N^{p} M^{a} \leqslant D
$$

Then in the notation of Lemma 3 we have

$$
G_{0} \leqslant \Delta_{p+q} G^{p} M^{\alpha}
$$

As in the proof of Theorem 1 we have

$$
\text { (4.5) } \begin{aligned}
V^{p} \sum\left|F_{1}(s, \psi)\right|^{\alpha} & \ll\left(G^{p} M^{\alpha} \Delta_{p+q}\right)^{1 / 2} B\left(2^{p+q} M^{q} N^{p}, U(h, \omega)\right) \\
& \ll G^{p / 2} M^{\alpha / 2} \Delta_{p+q}^{1 / 2}\left\{R^{1 / 2} M M^{\alpha / 2} N^{p / 2} l^{2}+R M M^{\alpha / 4} N^{p / 4} l^{2}+\right. \\
& \left.+R^{5 / 6} M^{\alpha / 6} N^{p / 6} D^{1 / 6} \Delta_{2}^{1 / 2} \Delta_{3}^{1 / 6} l^{3}\right\},
\end{aligned}
$$

where we have used (2.22). Hence

(4.6) $\quad B^{*}\left(D / N^{r}, U(h, \omega)\right) \ll R^{(\alpha-1) / \alpha} G^{x / 2 \alpha} V^{-3 / \alpha} \Delta_{y+\alpha}^{1 / 2 \alpha}\left\{R N^{p^{\prime}-r a} D^{a} l^{4}+\right.$

$$
\left.+R^{2} N^{(p \cdots r q) / 2} D^{\alpha / 2} l^{4}+R^{3 / 3} N^{(p \cdots r q) / 3} D^{(q+2) / 3} \Delta_{2}^{1 / 6} \Delta_{s}^{1 / 8} l^{6}\right\}^{1 / 2 q} .
$$

Lemman 2 applied to $F^{\prime \prime}(s, x)$ gives

$$
\begin{aligned}
R V^{r} \ll\left(\Delta_{r+1} Q^{r}\right)^{1 / 2}\left(R^{2 / 2} N^{r / 2} \log l+R N^{r / 4} l^{2}+\right. & \\
& \left.+R^{1 / 2} N^{r / 4} \Delta_{3}^{1 / 3} l^{9 / 4} \max _{n, \omega}\left\{B^{*}\left(D / N^{r}, U(h, \omega)\right)\right\}^{1 / 2}\right) .
\end{aligned}
$$


Comparing (4.7) with (4.6), we see that if

$$
\text { (4.8) } \quad V>e_{3} G^{1 / 2} N^{1 / 4}\left(\Delta_{r+1}\right)^{1 / 2 r} l^{2 / r} \text {, }
$$

where $c_{3}$ is an absolute constant, we have either

$$
R \ll Q^{r} V^{-2 r} N^{r} \Delta_{r+1} \log ^{2} l
$$

or

(4.10) $\quad R^{2}\left(V^{2} / G\right)^{2 \alpha r+q} N^{-\alpha r} \Delta_{3}^{-2 q} \Delta_{r+1}^{-2 q} \Delta_{p+\alpha}^{-1} l^{-9 q}$

$\ll R N^{p-r q} D^{q} l^{4}+R^{2} \cdot D^{(p-r q) / 2} D^{q / 2} l^{4}+R^{5 / 3} N^{(p-r q) / 3} D^{(q+1) / 3} A_{2}^{1 / 6} \Lambda_{3}^{1 / 3} l^{6}$

The condition (2.26) with a suitable $c_{2}$ implies (4.8). Also if (2.26) with at suitable $o_{2}$ and (4.10) hold then either
(4.11)
$R \ll\left(G V^{-2}\right)^{2 q r+p} N^{p} D^{\alpha} \Delta_{3}^{2 \alpha} \Delta_{r+1+1}^{2 q} 7^{q \alpha+1}$

or

$$
R \ll\left(G \nabla^{-2}\right)^{6 q r+3 p} N^{2 q r+p} D^{q+1} \Delta_{2}^{1 / 2} \Delta_{3}^{6 q+1+1} \Delta_{j++1}^{6 q} \Delta_{p++q^{3}}^{327 q+14} .
$$

This proves $(2.27)$ subject to $(2.26)$. The bound $(2.25)$ follows from $(2.27)$ and the formulae for $d_{\eta_{k}}(m)$.

5. Proof of Theorem 3, The first two assertions of Theorem 3 are easy consequences of Theorem 1. For (2.34) we use Theorem 2. Wo can absorb the fixed character $\chi$ into the coefficients $a(n)$ without increasing $G$, and take $Q=1, D=7$. In Theorem 2 we take $q \geqslant 2$; the case $q=1$ is different because an exponent of $T_{0}$ below becomes zero. We divide the range for $T$ in (2.27) into jntervals of length $T_{0}$ where $T_{0} \leqslant T$ and

$$
V \gg G^{1 / 2}\left(N^{q r+p} T_{0}^{\alpha+8}\right)^{1 /(8 q++4 p)}
$$

the constant necessary in (5.1) depending on $\varepsilon$. We use $\varepsilon$ for any exponent. which is $o(1)$, not necessarily the same at each occurrence. Then

$$
\begin{aligned}
R \ll\left(G N V^{-2}\right)^{r} T^{y+8} T_{0}^{-1}+\left(G V^{-2}\right)^{2 q q^{\prime}+p} N^{p} T^{1+8} T_{0}^{\alpha-1}+ & \\
& +\left(Q^{3} N V^{-6}\right)^{2 q r+p} T^{h+s} T_{0}^{\alpha+8}
\end{aligned}
$$

We choose $T_{0}$, if possible, to make the first two terms in (5.2) of the same order, that is

$$
T_{0}=\left(V^{2} G^{-1}\right)^{(2 Q r+2)-r) / Q} N^{(r-p) / q^{\prime}}
$$

The condition (5.1) requires

$$
\text { . (5.4) } V \geqslant G^{1 / 2} N^{r(\alpha+1+1+s)(4 \alpha r+2 p+2 r)}
$$

and (5.3) already implies $T_{0} \geqslant N^{r}$. The bound (5.2) becomes

(5.5) $\quad R T^{-1-B} \ll\left(G V^{-2}\right)^{(a q q+p)-r) / q} N^{(\alpha r+p-r) / q}+\left(G V^{-2}\right)^{4 q r+2 p+r} N^{2 q r+r}$.
The second term in $(5.5)$ is less than the first for

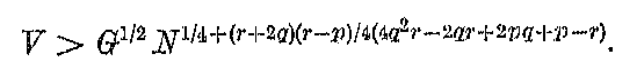

The condition (5.6) is stronger than (5.4).

We now suppose (2.33) holds, so that $T^{4}<N^{2+1}$. Substituting for $V$ from (2.29), we find that the first term in $(5.5)$ is less than $x^{2-2 a+s}$ for

$$
\alpha>\frac{3}{4}+\frac{p+q-r}{4(2 q r+p-q-r)},
$$

which simplitios to

$$
a>\frac{3}{4}+\frac{1}{4(4 r-3)}
$$

when $q=2, p=r-1$. These values of $p$ and $q$ malkes (5.6)

$$
\alpha>\frac{3}{4}+\frac{r+4}{4(16 r-3)}
$$

For $r \geqslant 3(5.9)$ is the stronger condition. We need not cheok $T_{0} \leqslant T$, for replacing $T_{0}$ by $T$ will reduce the second and third terms in (5.2), if $T_{0}>I$, and increase the tirst one to $X^{2 r(1-a)+s,}$, which is still less than $x^{2-2 a+8}$.

Alternatively we choose $T_{0}$ to equalise the first and third terms in (5.2), giving

(5.10) $\quad T_{0}=\left(V^{2} G^{-1}\right)^{(6 q r+3 p-r) /(q+1)} N^{-\left(2 q r^{2}+p-r\right) /(\alpha+1)}$.

We have $T_{0} \geq N^{r}$ for

(5.11) $\quad V \geqslant G^{1 / 2} X^{1 / 4+(r-p) / 1(6 q r+3 p-r)}$.

The condition (5.1) is satisfied for

$$
V<\alpha^{1 / 2} N^{\left.1 / 4+q(q)-2) / \alpha\left(2 q^{2} r-5 q r+2\right) q-2 p\right)}
$$

for $q>2$, or for

$$
V>G^{1 / 2} N^{\left.1 / d-q(r-p) / 4\left(s(q)-2 q^{2}++2 p\right)-p q\right)}
$$

when $q=1$ or 2 . Who choice of $T_{0}$ malkes (5.2)

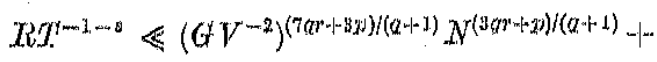

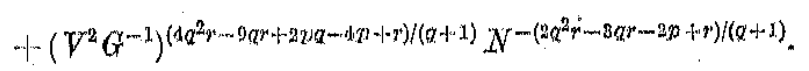

The second term in (5.14) is less than the lirst if (5.6) is false. Substituting for $V$ trom $(2.29)$ we tind that the first term in (5.14) is less than $T^{2-2 \alpha+8}$ 


$$
a>\frac{3}{4}+\frac{r-p+q+1}{4(6 q r+3 p-r-q-1)},
$$

a condition which implies (5.11). With $q=2, p=r-1$ (5.15) is

$$
\alpha>\frac{3}{4}+\frac{1}{14 r-6}
$$

which is $(2.35)$.

Exrata to Largo values of Dirialblet polynomiats

Equation(1.12): Insert factor $N^{1 / 4}$ in the final tiorm.

Equation(3.20): Right hand side shonld be $M(1-u) L(u, \underline{y})$.

Equation(4.5): should read $f=q_{2} / h_{1}$.

Equation(6.9): Replace $T_{1}$ by $F T_{1}$.

\section{Referencos}

[1] M. Forti and C. Viola, Density. estimates for the zeros of L-funotions, $\Lambda$ Arith. 23 (1973), pp. 379-391.

[2] M. N. Fuxley, On the difference between aonseative primes, Invent. Math. (1972), pp. 164:-170.

[3] - Large values of Diriohlet polynomials, Aota Arith. 24 (1973), pp. 329-3

[4] - Large values of Diriohlet polynomials II, Acta Arith. 25 (1.974), pp. 159-1

[5] M. Jutila, On a density thoorem of $H$. $t$. Montgomery for $L$-funotions, Anne Acad. Sci. Fenn. Series AT 520.

[6] H. L. Montgomery, Topios in Multiplicative Number Theory, Berlin-Heldelbes New York 1971.

Les volumes IV Volumes from IV Die Bände IV und Tомы IV й creдy. et suivants sont on are arailable die folgende sind za romue mo\%mo no

Ars Polona-Ruch, Krakowskie Przedmieście 7, 00-068 Warszawa (Poland)

Les volumes I-III Volumes I-III Die Bände I-III sind Tomm I-III можно sont d obtenir chez are available at zu beziehen durch полутить черев Johnson Reprint Corporation, 111 Fifth Ave., New York, N. Y. 